\title{
Bartonella species-induced prosthetic valve endocarditis associated with rapid progression of valvular stenosis
}

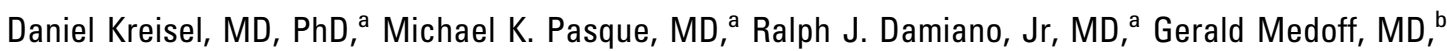 \\ Andrew Kates, MD, ${ }^{\mathrm{b}}$ Friederike H. Kreisel, MD, ${ }^{\mathrm{c}}$ and Jennifer S. Lawton, MD, ${ }^{a}$ St Louis, Mo
}

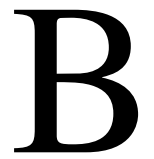

artonella species are fastidious gram-negative rods that comprise $2 \%$ of cases of blood culture-negative endocarditis. ${ }^{1}$ Although most cases of Bartonella species-induced endocarditis affect native valves, the few case reports describing Bartonella species-induced prosthetic valve endocarditis are characterized by vegetations and valvular perforations, leading to valvular insufficiency. ${ }^{1-3}$ Here we report 2 cases of Bartonella species-induced endocarditis affecting prosthetic mitral valves, which were associated with rapid progression of mitral stenosis and development of heart failure.

\section{Clinical Summary}

PATIENT 1. A 36-year-old man with a history of alcohol and intravenous drug abuse had undergone mitral valve replacement with a porcine valve for Staphylococcus aureus-induced bacterial endocarditis. An echocardiogram obtained 9 months postoperatively showed a mitral valve area of $2 \mathrm{~cm}^{2}$ and a mean mitral valve gradient of $4 \mathrm{~mm} \mathrm{Hg}$. Four months later, he presented with exertional dyspnea and acute renal failure, with a serum creatinine level of $6.3 \mathrm{mg} / \mathrm{dL}$. An echocardiogram demonstrated a thickened prosthetic valve and a mean mitral valve gradient of $14 \mathrm{~mm} \mathrm{Hg}$. An indirect fluorescent antibody test revealed increased serum IgG titers to Bartonella henselae (1:2048) and Bartonella quintana $(1: 1024)$. He was started on intravenous antibiotics. Further work-up revealed splenomegaly and mediastinal lymphadenopathy. A percutaneous renal biopsy revealed crescentic glomerulonephritis. His serum creatinine level improved to $3 \mathrm{mg} / \mathrm{dL}$ after treatment with corticosteroids. Over the next 2 weeks, the patient experienced rapidly progressive congestive heart failure. Echocardiography showed worsening prosthetic mitral valvular stenosis with a severely calcified prosthetic valve. The mitral valve area was $0.7 \mathrm{~cm}^{2}$, and the mean mitral valve gradient was $24 \mathrm{~mm} \mathrm{Hg}$. The patient underwent a redo mitral valve replacement with a mechanical valve (Figure 1, A). Rod-shaped bacterial organisms were identified in the excised valvular tissue (Figure $1, B$ ). Valve

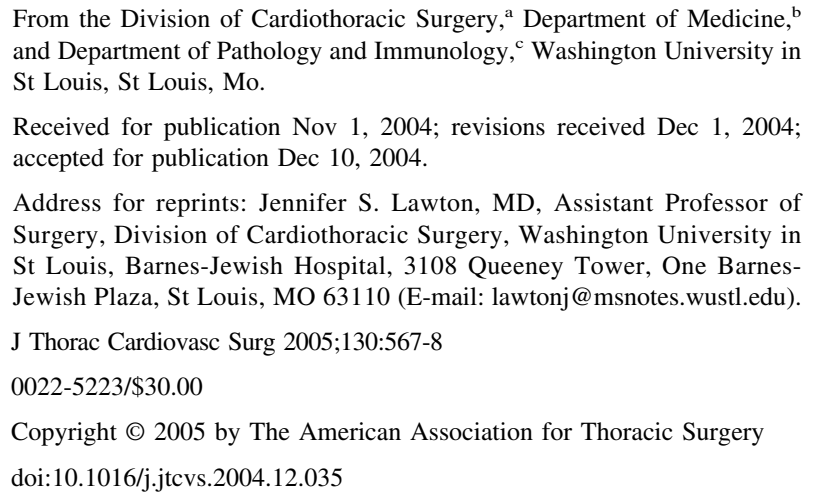

tissue and mediastinal lymph nodes were positive for $B$ henselae, as determined by means of polymerase chain reaction. The patient had an uneventful postoperative course, his serum creatinine level normalized, and he was discharged on oral antibiotics.

PATIENT 2. A 76-year-old man had undergone mitral and aortic valve replacement with porcine valves for severe mitral and aortic stenosis. Sixteen months postoperatively, the patient had low-grade fevers and fatigue. An echocardiogram revealed mild mitral stenosis with a mitral valve area of $2 \mathrm{~cm}^{2}$ and a mitral valve gradient of $7 \mathrm{~mm}$ $\mathrm{Hg}$. The aortic valvular prosthesis was normal. Work-up revealed increased serum IgG titers to $B$ henselae (1:1024) and $B$ quintana (1:1024), and he was started on intravenous antibiotics for prosthetic valve endocarditis. The patient experienced renal failure, with serum creatinine levels as high as $8 \mathrm{mg} / \mathrm{dL}$. A percutaneous renal biopsy revealed crescentic glomerulonephritis, and the patient was started on corticosteroids. Over the ensuing 8 weeks, the patient had worsening congestive heart failure. Serial echocardiograms demonstrated rapidly progressive mitral stenosis, with severe thickening of the mitral valve prosthesis. The mean mitral valve gradient was measured at $25 \mathrm{~mm}$ $\mathrm{Hg}$, and the mitral valve area was $1 \mathrm{~cm}^{2} 8$ weeks after his initial presentation. The aortic valve prosthesis was only mildly thickened. The patient declined surgical intervention and died of this complication.

\section{Discussion}

$B$ quintana and $B$ henselae are the 2 main pathogens for blood culture-negative Bartonella species-induced endocarditis. This infection has been mostly observed in homeless patients and alcoholics. Although patient 1 was an alcoholic, patient 2 had no known risk factors for infection with Bartonella species. Only a few cases of Bartonella species-induced prosthetic valve endocarditis have been described in the literature. One patient presented with Bartonella species-induced endocarditis of a prosthetic aortic valve 8 years after valve implantation. ${ }^{2}$ The patient was found to have vegetations and paravalvular regurgitation and underwent valve replacement after failure of antibiotic treatment. Another patient, who was diagnosed with $B$ henselae-induced endocarditis 3 years after aortic valve replacement, was cured with long-term antibiotics without requiring surgical intervention. ${ }^{3}$ Echocardiographic findings at presentation were limited to nodular thickening of the anterior cusp of the aortic bioprosthesis. Interestingly, a recent study demonstrated the presence of intact bacteria in extracellular locations associated with extensive fibrosis and numerous calcifications in native valves affected by Bartonella species-induced endocarditis. ${ }^{4}$ Prolonged subclinical infection with Bartonella species was thought to lead to chronic inflammatory changes. Similarly, bacterial antigenic material has been detected in calcified nodules of a native aortic valve in a 6-year-old boy, implicating $B$ henselae in the pathogenesis of calcific aortic stenosis. ${ }^{5}$ 

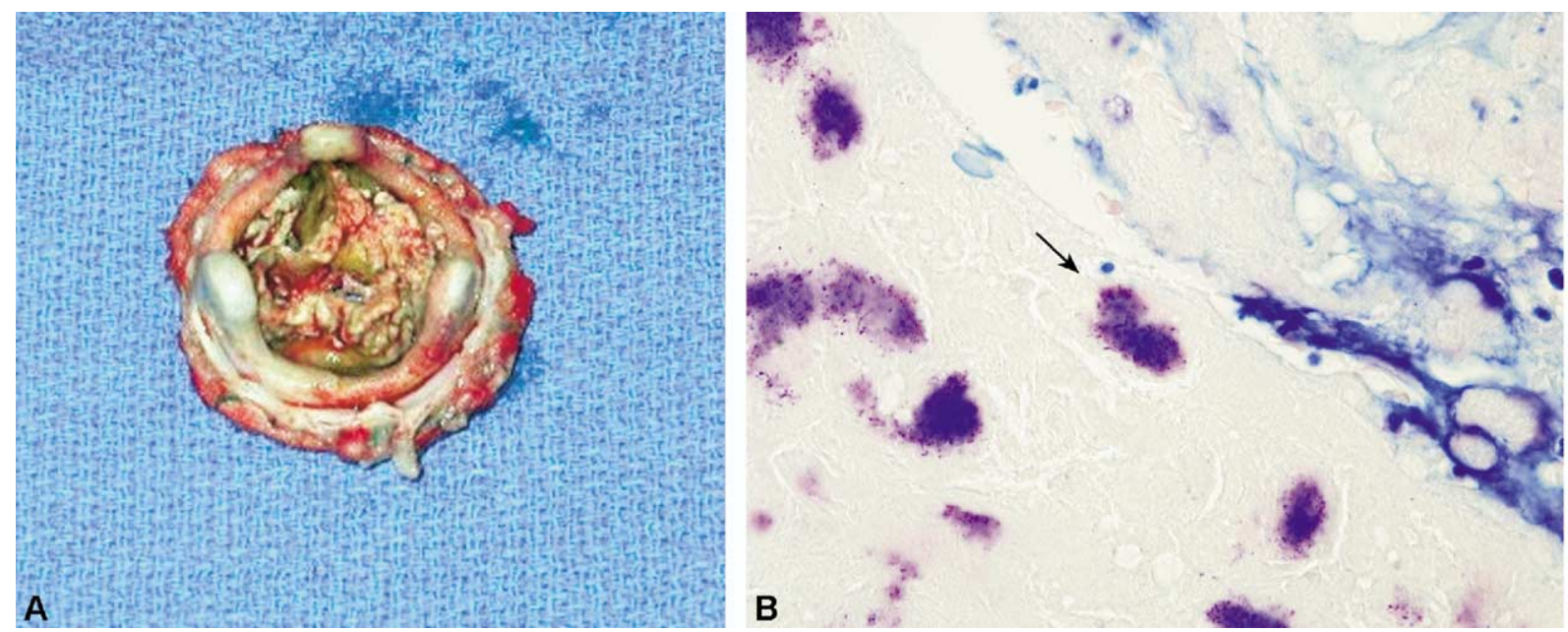

Figure 1. A, Hancock II porcine mitral valve destroyed by Bartonella species-induced endocarditis. B, A Giemsa stain (100X magnification) shows colonies of small rod-shaped bacterial organisms (arrow) embedded in necrotic debris.

To our knowledge, this is the first report of Bartonella speciesinduced prosthetic valve endocarditis associated with rapid progression of valvular stenosis and deterioration in hemodynamics. Both patients had renal failure caused by immune complex-mediated glomerulonephritis. Expeditious valvular replacement in addition to antibiotic treatment is warranted for this rare clinical condition.

\section{References}

1. Lamas CC, Eykyn SJ. Blood culture negative endocarditis: analysis of 63 cases presenting over 25 years. Heart. 2003;89:258-62.
2. Klein JL, Nair SK, Harrison TG, Fry NK, Friedland JS. Prosthetic valve endocarditis caused by Bartonella quintana. Emerg Infect Dis. 2002;8: 202-3.

3. Lesprit P, Noel V, Chazouilleres P, Brun-Buisson C, Deforges L. Cure of Bartonella endocarditis of a prosthetic aortic valve without surgery: value of serologic follow-up. Clin Microb Infect Dis. 2003; 9:239-41.

4. Lepidi H, Fournier PE, Raoult D. Quantitative analysis of valvular lesions during Bartonella endocarditis. Am J Clin Pathol. 2000;114: 880-9.

5. Ghidoni JJ. Role of Bartonella henselae endocarditis in the nucleation of aortic valvular calcification. Ann Thorac Surg. 2004;77: 704-6. 